\title{
Fibular lengthening by Ilizarov method secondary to shortening by osteochondroma of distal tibia
}

\author{
A. J. Johnston · C. T. Andrews
}

Received: 9 October 2007 / Accepted: 10 December 2007/Published online: 11 January 2008

(C) Springer-Verlag 2008

\begin{abstract}
Osteochondroma is the most common benign bone tumour. They most commonly affect the long tubular bones and almost half of osteochondromata are found around the knee. Osteochondroma arising from the distal metaphysis of the tibia typically result in a valgus deformity of the ankle joint secondary to relative shortening of the fibula. This case describes the use of Ilizarov technique for fibular lengthening following excision of a distal tibial osteochondroma. A 12-year-old girl presented with a 3-year history of a large swelling affecting the lateral aspect of the right distal tibia. Plain radiographs confirmed a large sessile osteochondroma arising from the postero-lateral aspect of the distal tibia with deformity of the fibula and $15 \mathrm{~mm}$ of fibular shortening. The patient underwent excision through a postero-lateral approach and subsequent fibular lengthening by Ilizarov technique. The patient made excellent recovery with removal of frame after 21 weeks and had made a full recovery with normal ankle function by 6 months. The Ilizarov method is a commonly accepted method of performing distraction osteogenesis for limb inequalities; however, this is mainly for the tibia, femur and humerus. We are unaware of any previous cases using the Ilizarov method for fibular lengthening. This case demonstrates the success of the Ilizarov method in restoring both fibular length and normal ankle anatomy.
\end{abstract}

\footnotetext{
A. J. Johnston ( $\square)$

Musgrave Park Hospital, Belfast BT9 7JB, UK

e-mail: andyjohnston1975@hotmail.co.uk

C. T. Andrews

Musgrave Park Hospital, Belfast BT9 7JB, UK
}

Keywords Fibular shortening - Osteochondroma . Deformity · Ilizarov

\section{Introduction}

Osteochondroma is the most common benign bone tumour. They most commonly affect the long tubular bones such as the femur, humerus, radius, tibia and fibula, almost half of osteochondromas are found around the knee, in either the distal femur or the proximal tibia.

Osteochondromata are located adjacent to growth plates and they develop away from the growth plate. They are affected by and respond to various growth factors and hormones in the same manner as epiphyseal growth plates, therefore osteochondromas enlarge during the growth of the skeletal system and the growth ceases at skeletal maturity.

In less than $1 \%$ of solitary osteochondromas, malignant degeneration of the cartilage cap into secondary chondrosarcoma has been described and is usually associated with new onset of growth of the lesion, new onset of pain, or rapid growth of the lesion.

Asymptomatic lesions require no treatment and can be observed with initial radiographs and subsequent clinical examination. Patients may experience pain secondary to a direct mass effect on the overlying soft tissue, mechanical trauma or an inflamed bursa overlying the lesion. Excision is the treatment of choice in this situation.

Osteochondroma arising from the distal metaphysis of the tibia is previously described within the literature. This typically results in a valgus deformity of the ankle joint secondary to relative shortening of the fibula [3-6, 8].

We describe a case of distal tibial osteochondroma treated with excision and fibular lengthening by Ilizarov technique. 


\section{History}

A 12-year-old girl presented with a 3-year history of a large swelling affecting the lateral aspect of her right distal tibia.

She was not experiencing any associated pain or restriction of normal activities. She walked without a limp; examination confirmed a hard, non-tender lump overlying the lateral aspect of the right distal tibia. There was no hind-foot deformity and she had a full range of movement of the ankle joint, there was no neurovascular deficit. She had no other associated swellings.

Both patient and parents had concerns over the cosmetic appearance of the limb and potential long-term consequences that may arise.

Plain antero-posterior and lateral radiographs of the right tibia and ankle joint confirmed a large sessile osteochondroma on the postero-lateral aspect of the tibia. It caused resultant pressure problems in the fibula, with deformity and $15 \mathrm{~mm}$ shortening of the fibula. Both talocrural angle and the tibial plafond inclination were measured at $2^{\circ}$ and $6^{\circ}$ respectively; this provided a method to observe change following completion of the lengthening (Fig. 1).
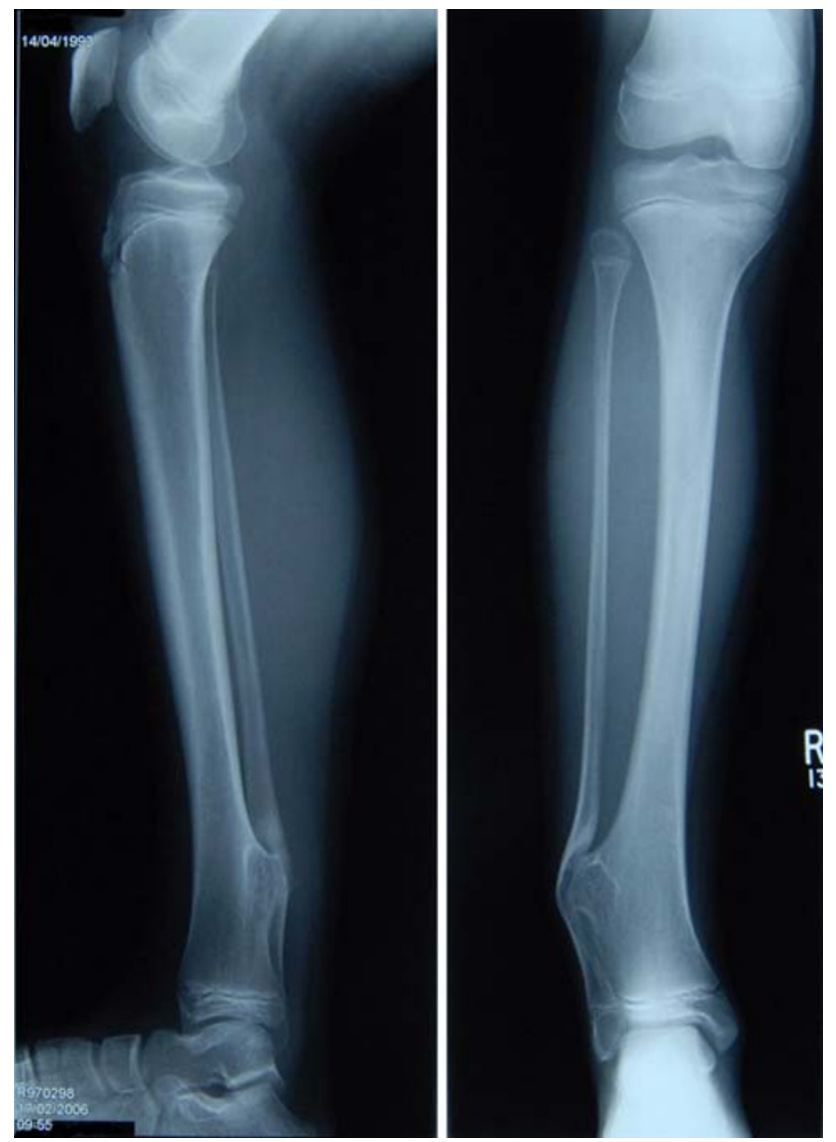

Fig. 1 Preoperative anteroposterior and lateral radiographs right tibia
Although the patient had no significant deformity in the hind foot at presentation it was felt surgical excision was the treatment of choice. If left untreated the patient would develop the characteristic valgus deformity of the hindfoot and associated ankle instability secondary to the relative shortening of the fibula.

The patient underwent excision of a sessile tibial osteochondroma through a postero-lateral approach and Ilizarov lengthening of distal fibula.

An Ilizarov frame was constructed with two $140 \mathrm{~mm}$ Ilizarov rings. The proximal ring was fixed with two proximal tibial wires and the distal ring with three wires, including a fibular wire. Two $4 \mathrm{~mm}$ half-pins were used in the fibula, one epiphyseal and one metaphyseal. A percutaneous fibular osteotomy, with drill and osteotome, of the distal quarter of the fibula was performed (Figs. 2, 3).

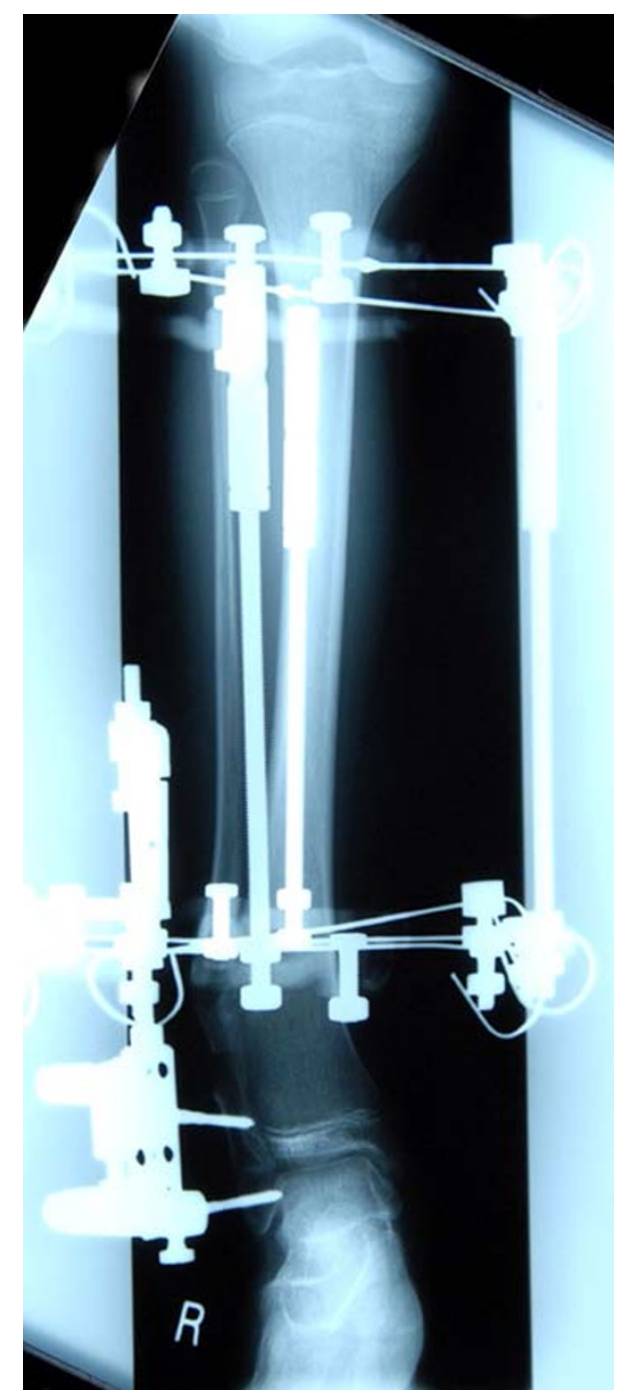

Fig. 2 AP radiograph tibia following completion of fibular lengthening 


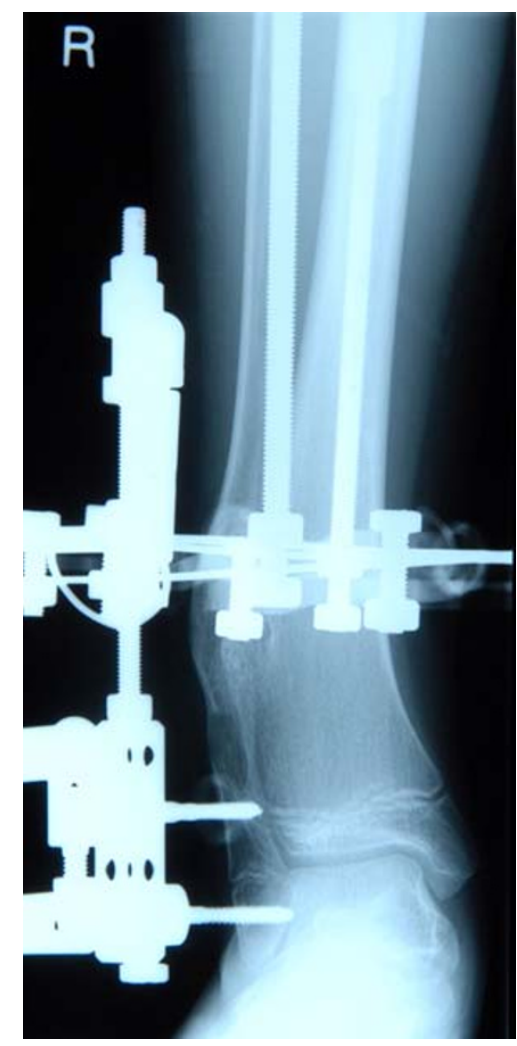

Fig. 3 AP radiograph showing consolidation of bone regenerate

Post-operative care included a pin site care regime, a thermoplastic foot splint, full-weight-bearing mobilisation and regular physiotherapy. Lengthening commenced after a 5-day latent period with $1 \mathrm{~mm}$ per day distraction (divided into four $0.25 \mathrm{~mm}$ parts) for 12 days.

Histology confirmed a benign osteochondroma with no evidence of malignancy.

Post-operatively the patient made an excellent recovery with no complications and underwent removal of Ilizarov frame after 21 weeks.

Radiologically, following the fibular lengthening, the talo-crural angle measured $11^{\circ}$ compared to $2^{\circ}$ on preoperative films. The angle of inclination of the tibial plafond was minimally changed measuring $4^{\circ}$.

At 6-month follow-up she had made a full recovery with normal functions of the ankle joint (Figs. 4, 5).

\section{Discussion}

Osteochondromata are the most common benign bone tumour and usually follow a predictable course. Malignant change to chondrosarcoma is rare; the more common complication is nerve compression or skeletal deformity producing local pressure symptoms. Osteochondromata affecting the distal tibia may result in anatomical

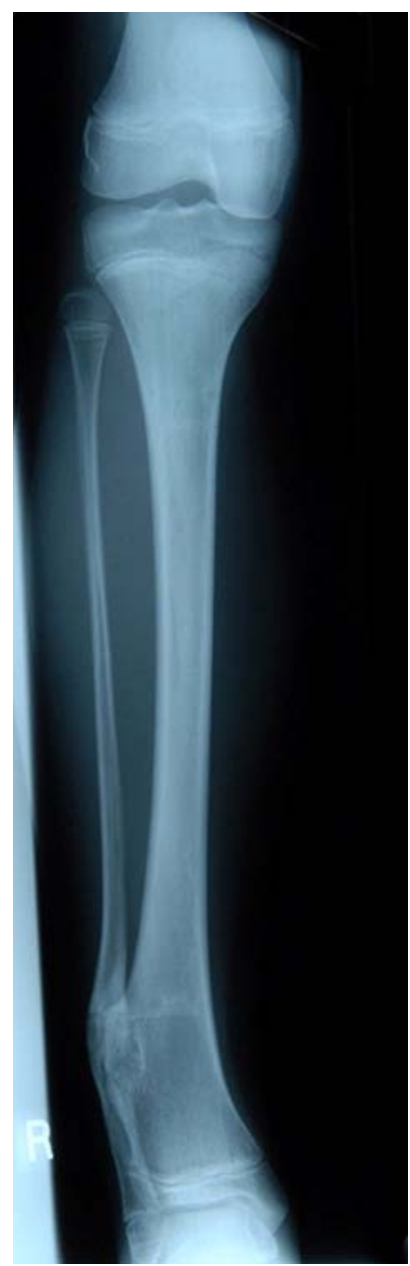

Fig. 4 AP radiograph following removal of Ilizarov frame

abnormalities of the ankle resulting in reduced motion, bowing of the fibula, fibular shortening, valgus deformity of distal tibial epiphysis and distal tibiofibular diastasis. Subsequently a combination of these deformities may predispose the patient to long-term degenerative disease of the ankle joint $[8,9]$.

It has been demonstrated that the lateral malleolus contributes to stability of the ankle, maintaining the talus in its proper anatomical and biomechanical position. The importance of correct fibular length has been demonstrated with increased contact pressures within the ankle joint following $2 \mathrm{~mm}$ of shortening of the lateral malleolus [10-12].

Although the patient in this case had no impairment of ankle function, the decision to treat the lesion non-operatively may result in the development of further ankle deformity and predispose the patient to tibiotalar arthritis or ankle instability and recurrent injury [3, 8].

Fibular lengthening has been described in similar cases by distraction, bone-grafting and internal fixation and a combination of external and internal fixation [7, 8]. 


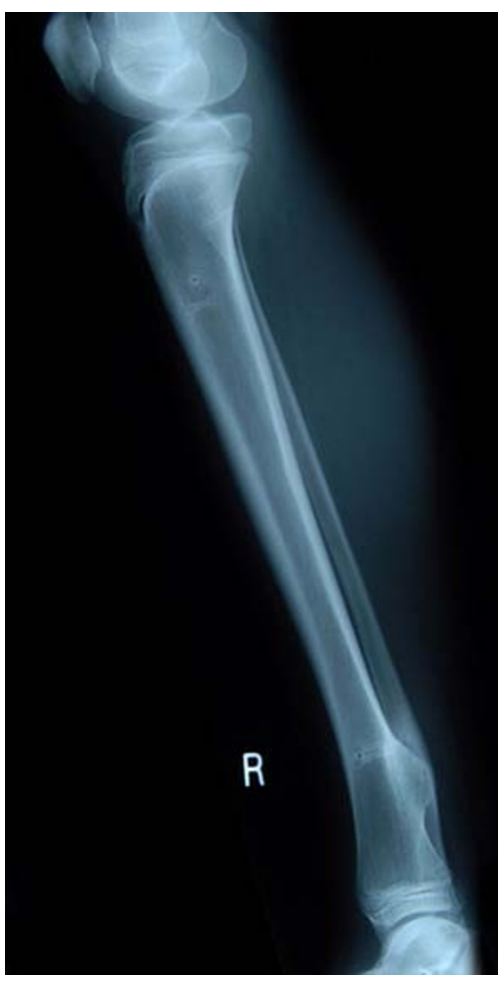

Fig. 5 Lateral radiograph following removal of Ilizarov frame

This case demonstrates the use of the Ilizarov method to regain fibular length following excision of a distal tibial deforming osteochondroma. The Ilizarov method is commonly described for lengthening of other long bones mainly tibia, femur and humerus [1,2]. We are not aware of any similar cases in the literature were Ilizarov has been used exclusively for fibular lengthening.

This method avoids the need for soft tissue stripping, comorbidity associated with bone grafting, the increased risk of infection when used in combination with internal fixation and need for post-operative immobilisation. In this case the patient was able to mobilise immediately fullyweight-bearing and commence physiotherapy to maintain ankle function.
This case highlights the need for consideration of early intervention to excise distal tibial ostechondromata before subsequent fibular and hind-foot deformities arise. It also shows the Ilizarov method as a successful means of restoring both fibular length and normal ankle anatomy with excellent clinical outcome.

\section{References}

1. Aaron AD, Eilert RE (1996) Results of the Wagner and Ilizarov methods of limb-lengthening. J Bone Joint Surg 78-A(1):20-29

2. Atar D, Lehman WB, Grant AD, Strongwater A, Frankel V, Golyakhovsky V (1991) Treatment of complex limb deformities in children with the Ilizarov technique. Orthopaedics 14(9):961967

3. Chin KR, Kharrazi D, Miller BS, Mankin HJ, Gebhardt MC (2000) Osteochondromas of the distal aspect of the tibia or fibula: natural history and treatment. J Bone Joint Surg 82-A(9):12691277

4. Danielsson LG, El-Haddad I, Quadros O (1990) Distal tibial osteochondroma deforming the fibula. Acta Orthop Scand 61(5):469-470

5. Durak K, Bilgen O, Kaleli T, Aydinli U (1996) Distal fibula resection in osteochondroma. J Int Med Res 24:381-386

6. Gupte CM, DasGupta R, Beverly MC (2003) The transfibular approach for distal tibial osteochondroma: an alternative technique for excision. J Foot Ankle Surg 42(2):95-98

7. Rozburch SR, DiPaola M, Blyakher A (2002) Fibula lengthening using a modified Ilizarov method. Orthopaedics 25(11):12411244

8. Snearly WN, Peterson HA (1989) Management of ankle deformities in multiple hereditary osteochondroma. J Pediatr Orthop 9(4):427-432

9. Spatz DK, Guille JT, Kumar SJ (1997) Distal tibiofibular diastasis secondary to osteochondroma in a child. Clin Orthop Relat Res 345:195-197

10. Thordarson DB, Motamed S, Hedman T, Ebramzadeh E, Bakshian S (1997) The effect of fibular malreduction on contact pressures in an ankle fracture malunion model. J Bone Joint Surg 79:1808-1815

11. Yablon IG, Heller FG, Shouse L (1977) The key role of the lateral malleolus in displaced fractures of the ankle. J Bone Joint Surg 59-A(2):169-173

12. Yadav SS (1981) Ankle stability after resection of the distal third of the fibula for giant-cell lesions: report of two cases. Clin Orthop Relat Res 155:105-107 